\title{
No sin la clínica
}

\section{Not without the clinic}

\section{Mario Kelman}

\section{RESUMEN:}

El presente escrito muestra dos cuestiones interrelacionados.

En primer lugar, interrogar la actualidad de la ley nacional de salud mental a través de la experiencia previa sostenida y compartida como practicante de la clínica, en el ámbito público de la salud mental en la Provincia de Santa Fe.

En segundo lugar, y como consecuencia inherente y decantada en otro tiempo lógico respecto de la experiencia -momento de paso y reescritura- abrir la interrogación sobre la intersección del discurso analítico y lo político.

\section{Palabras clave:}

Salud - Mental - Ley - clínica psicoanálisis

\section{SUMMARY:}

The following article poses two interrelated matters.

In the first place, inquiring about the current relevance of the National Mental Health Law through sustained and shared previous experience as a practitioner of the clinc in the public sector of mental health in the Santa Fe province. In the second place, and as a consequence, both inherent and established in another logical time period with respect to experience -moment of pass and rewriting-, inquiring about the intersection of analityc and political discourse

\section{WORD PAD:}

Healt - Mental - Law - Clinic -

Psychoanalysis 

Hitos

Sanción de la Ley Provincial I0772/9 I y de la Ley Nacional 26657/IO

La sanción de la Ley Nacional de Salud Mental 26657/10 se presenta como un cambio de paradigma respecto de quién padece una afección psíquica. Implica el pasaje de una lógica que lo considera como objeto de tutela del Estado, a otra lógica que lo supone un sujeto de derecho.

Si bien la adquisición de derechos siempre es una buena noticia, es oportuno y pertinente el reconocimiento de que el cambio referido ya existía en la Provincia de Santa Fe. No sólo en los enunciados de la Ley 10772/91, sino en su incorporación en la experiencia viva, el uso y aplicación por parte de la comunidad de practicantes de la clínica concernidos, que fueron parte de una histórica reforma de salud mental implementada en la Colonia Psiquiátrica "Irigoyen Freyre" de la localidad de Oliveros, que se irradió al conjunto de instituciones de la provincia. Lamentablemente hay muy pocos testimonios escritos acerca de la reforma de Salud Mental en la Provincia de Santa Fe, que estableció una gran diferencia respecto de la mayoría de las provincias del país. (Aguirre, 2014)

La Ley 10772 sancionada en 1991, establece que la asistencia de las personas que padecen un sufrimiento psíquico tiene el estatuto de un derecho. Prioriza los tratamientos que menos restrinjan la libertad de la persona o lo alejen de su núcleo social, conservando la decisión de aceptar o rechazar el tratamiento ofrecido. Se dispone el derecho de ser escuchado y el consentimiento informado de la terapéutica propuesta. Se establecen criterios de internación voluntaria y no voluntaria, con las debidas garantías de derecho, cuyo peso de gravedad radica en el cuidado de una persona que está atravesando un momento de alta sensibilidad o crisis. Se establece el instituto de Departamentos Jurídicos en Servicios e Instituciones de Salud Mental, que garantizan el respeto de los derechos y garantías personales y DDHH; así como la habilitación de medios terapéuticos, que constituyen una apertura a la producción de dispositivos alternativos.

La experiencia de Santa Fe, incluso excede los términos de la ley 26657, en la propuesta del artículo 22 de la ley provincial, que habilita la sustanciación de Juntas Interdisciplinarias de Dictámenes en el ámbito del Ministerio de Salud y Medio Ambiente.

Se trata de un instrumento adecuado y efectivo, con mayor jerarquía jurídica y superador de los Dictámenes del Gabinete Forense; que por otra parte, evita la judicialización de los casos. La presente mención constituye una invitación al lector, a interiorizarse sobre el tema.

El artículo 22 mereció dos reglamentaciones. El Decreto 2155/2007 reglamenta la ley 10772/91 con su forma vigente. Pero existe el antecedente de la Resolución 660 del MSyMA de la Provincia de Santa Fe, emitido el 7 de abril de 2003, redactada por una Comisión integrada por las psicoanalistas Ana Ruth Najles y Susana Toté, y el Dr. Mariano Ciaffardini y su equipo de colaboradores. Dicha Reglamentación hizo lugar a una experiencia de Juntas Especiales que se sostuvo con consecuencias fecundas entre los años 2003 y 2007.

Esta versión otorgaba una participación en igualdad de jerarquía, al sector de enfermería; garantizaba una amplia y simple accesibilidad jurídica; no sólo a 
jueces, sino también a instituciones, familiares y personas interesadas. Instituía un Comité Interdisciplinario Permanente y la integración de Juntas Ad Hoc -con el concurso de los Colegios Profesionales- para resolver caso por caso; resultando así, dos instancias de revisión y control recíproco que otorga garantías de Derecho al procedimiento.

Las Juntas Ad Hoc debían emitir Dictamen en un plazo máximo de 30 días, verificando los requisitos legales y clínicos de una internación; la existencia de condiciones de externación y continuidad de tratamiento ambulatorio. Debían denunciar ante la Defensoría de Menores e Incapaces los casos de agravamiento de las condiciones de privación de libertad; y si estuviera en el marco de una medida de seguridad, dar conocimiento al Juzgado que la dispuso. Dar igual aviso cuando el lapso de la internación dispuesta con motivo de la aplicación de una medida de seguridad se prolongara más allá de la mitad del monto de la pena que le hubiere correspondido al autor, de no haberse decretado su inimputabilidad. Por último, controlar el funcionamiento del Comité Interdisciplinario Permanente en el caso.

Las Juntas Interdisciplinarias de Dictámenes, junto al trabajo decidido de abogados en sus respectivos Departamentos Jurídicos en Servicios e Instituciones de Salud Mental, enmarcados en el artículo 24 de la ley 10772/91; han sido depositarios eficaces de la aplicación de los DDHH y los Derechos de las Personas en el campo de la Salud Mental.

La conclusión provisoria es que la ley 26657 si bien pudo significar un cambio en el país, no ha tenido el mismo efecto en la experiencia practicante en el campo de la salud mental de la provincia de Santa
Fe, donde los cambios fueron anticipados y ya incorporados en una experiencia propia.

\section{El estatuTo DE LA Ley}

\section{Y LA EXPERIENCIA VIVA}

Si se figura la impronta de un trazo en un plano topológico, advertimos que éste no delimita un solo espacio, sino dos espacios. De un lado escribimos S1, significante Uno, que nos remite a la Ley, el Ideal, el Nombre del Padre, el campo del lenguaje.

Del otro lado, escribimos "a"; en este contexto, lo viviente, lo sensible, el pathos.

El discurso del Derecho aproxima lo viviente a la norma. El discurso del Derecho aprehende la vida y la inscribe en lo simbólico. Pero vida y norma no coinciden ni armonizan, coexisten en los encuentros y desencuentros, incluso en el conflicto. La Ley se formula necesariamente como Universal y la naturaleza de lo vivo, lleva consigo la impronta de la singularidad de cada viviente. Lacan menciona en el Seminario La Identificación (Lacan, 1962-63) esta hiancia de estructura, que nombra como Lexis y Phasis, disyunción entre la función y la existencia.

A su vez, la sanción de una ley tampoco delimita un solo espacio, sino dos: dentro y fuera de la Ley. Nancy -citado por Agamben- define que la Ley tiene estructura de Bando (Agamben, 2002). El Bando refleja la dualidad de espacios de la ley, en tanto refiere tanto al emblema del soberano y su proclama, como a la exterioridad de su dominio territorial. De allí el bandolero, quién es puesto fuera de la ley y expulsado del ámbito comunitario del soberano. 
Para el Derecho, el fuera de la Ley es la transgresión. Es un afuera siempre en relación con los efectos de la aplicación y sanción de la Ley. El axioma Dura lex, sed lex afirma que no hay espacio humano que escape a la aplicación de la ley.

El Código Civil es un claro ejemplo que insta al recubrimiento de la vida por la norma. Basta relevar la coincidencia del índice con el trayecto que se extiende desde antes del nacimiento hasta después de la muerte.

La perspectiva kantiana del Derecho presenta el extremo de subsumir la vida en una norma llevada a un estado de imperativo universal, el Logos; pero forcluyendo el pathos, lo sensible viviente, en imparidad con la ley.

La experiencia analítica vela por la dignidad de lo viviente y aporta una noción de fuera de norma diferente a la del Derecho. El hiato entre el viviente y la ley es necesario para que tenga lugar la experiencia singular y el sujeto responsable.

En la experiencia analítica aprehendemos su efecto en la separación y distancia entre el Ideal y el objeto "a”. Cuando el Ideal se torna absoluto, y no admite distancia ni diferencia, adquiere una consistencia que abole el advenimiento del sujeto deseante singular.

Freud anuda cuerpo y vida, cuando anuda la pulsión de muerte con la repetición, repetición cuyo modelo es el retorno del cuerpo vivo a lo inanimado. Lacan avanza un paso respecto de Freud, y separa cuerpo y vida. Ubica el cuerpo en lo imaginario y la vida en lo real. Para Lacan, lo viviente escapa a lo imaginario y a lo simbólico. La vida y la muerte constituyen un misterio opaco. No obstante, sin reunirse, Real, Simbólico e Imaginario se articulan, se anudan.
Lo Simbólico no recubre lo Real en su totalidad, ni todo lo Real está articulado a lo Simbólico. En su última enseñanza, Lacan presenta un real sin ley. Real sin orden ni jerarquía, respecto del cual se verifica la respuesta ética de cada ser hablante; es decir, su sinthoma. A diferencia de la interpretación kantiana del Derecho, la experiencia analítica aloja la hiancia entre el pathos y la norma. Hay la norma y hay la vida singular, lo fuera de norma que aloja la experiencia ética singular de lo humano y la decisión que especifica la responsabilidad subjetiva.

Encontramos así, un fuera de norma que refiere a lo indeterminado, lo indecidible y que abre a la dimensión de una existencia posible. Es un fuera de norma del ser humano hablante.

La ley hace lugar y habilita la experiencia de lo humano, cada vez... También hay en lo real lo que preexiste a la espera de recibir la marca de lo simbólico, sostenido en la consistencia imaginaria. Se trata ya de un saber en lo real allí, que no es el saber inconsciente.

En consecuencia, el debate no se agota en el estudio comparativo de los enunciados eruditos de las leyes 10772 y 26657, sino cotejando experiencias que le ex-sisten.

Una breve digresión sobre el término ex-sistencia, sin entrar en desarrollos que exceden el alcance de este trabajo.

Lacan distingue el ser de la existencia y deconstruye este último término en la huella de Heidegger, modificándolo como ex-sistencia. Se pueden hallar referencias específicas en el Seminario sobre la letra robada (Lacan, 1956) y en el Seminario RSI (Lacan, 1974-75). En este contexto, "ex-sistencia" alude a una referencia topológica que implica la articulación, en 
diferente nivel, de elementos heterogéneos y discretos, que articulados no pierde su condición de discreción y heterogeneidad.

La experiencia sostenida por la comunidad de practicantes en Santa Fe tuvo varios sesgos interesantes.

En primer lugar, existió una comunidad de practicantes de la clínica que encarnaron las políticas públicas sostenidas en la construcción de una transferencia de trabajo.

En segundo lugar, dicha comunidad de practicantes adherían a la clínica psicoanalítica y a la clínica del fármaco -psiquiatría y neurociencia- encuadrada en un uso ético de los medicamentos, aceptando otros saberes concernidos, manteniendo la responsabilidad acerca de la dirección de cada cura, a través de la designación de terapeuta responsable.

Si consideramos una definición amplia de la clínica como respuesta al sufrimiento humano, se verifica en la práctica, la existencia de una posición clínica no sólo de la Medicina y el Psicoanálisis, sino del Derecho, del Trabajo Social, de Enfermería, etc; en tanto sensibles y concernidos por la interpelación del sufrimiento psíquico.

Cabe la interrogación sobre qué experiencia ha resultado a partir de la sanción de la ley 26657, tomando nota que no se produjeron políticas públicas acordes y suficientes a su aplicación, ni encontramos testimonios de una experiencia sostenida en una transferencia de trabajo.

No obstante, hay cuestiones en el plano enunciativo de la ley que tuvieron consecuencias. Tomaremos como referencias discursivas intercambios mantenidos por el autor con co-autores del texto de la ley 26657 y el informe anual sobre DDHH del CELS, correspondiente al año 2012.
El informe de 2012 del CELS retoma investigaciones anteriores, concluyendo en el establecimiento y denuncia de las violaciones generalizadas de los $\mathrm{DDHH}$ de las personas que tienen algún padecimiento psíquico.

Se denominan ahora usuarios de salud mental, asignándoles la categoría de un colectivo.

Coincidiremos en reconocer la historia de segregaciones que tienen larga data, tanto dentro como fuera de nuestro país, donde la locura es situada en el lugar de la excepción, continuando una larga serie de enfermedades malditas e impronunciables, tales como lepra, sífilis, cáncer, HIV; cuyo estudio Foucault nos ha brindado un minucioso testimonio. A las cuales podríamos agregar otras figuras más actuales de la exclusión social, no sólo vagos, maleantes sino sujetos precarizados, refugiados $\mathrm{y}$ migrantes.

La segregación es un hecho trágico que halla su peor manifestación en el Estado nazi, y tiene una complejidad que alcanza a todo hecho humano, en tanto interviene el lenguaje. Por razones de extensión, no avanzaremos en el desarrollo de su lógica. Pero nos limitaremos a señalar que la exclusión hace pareja con la inclusión. No hay de la una sin de la otra, y ambas generan y sostienen una aporía irresoluble, sin salida. La inclusión como vía de construcción de un Todo encuentra su límite inexorablemente en lo imposible. La inclusión traza su recorte e inevitablemente reproduce la exclusión. Por último, ¿qué lugar, qué función, qué autoridad se abroga quién pretende situarse como fiel de la balanza de inclusión o exclusión?

Un horizonte más fecundo pueda orientarse en otra lógica, la producción de 
ex-sistencia, uno por uno, construcción singular que conlleva el despliegue de la naturaleza propia, construyendo exterioridad. Es la vía del sinthoma. Se trata de acompañar la construcción posible a partir de lo que cuenta cada uno, responsabilizándose de ello, uno por uno, sin hacer un todo. Designar un colectivo de usuarios ¿no es un modo de segregación?

La segregación excede el campo de la salud mental.

El informe del CELS tiene una lógica dual, donde contrapone políticas hegemónicas con la construcción de políticas alternativas y reivindicativas. Denuncia críticamente el discurso médico-psiquiátrico, psicológico y psicoanalítico a cuenta de la producción de un modelo hegemónico y dominante que contribuye a la consolidación de prejuicios sociales, la disciplina y la segregación. Finalmente, homologa el padecimiento en salud mental con una discapacidad y propone su abordaje en los términos que determina la Convención de Derechos de Personas con Discapacidad de la ONU, referenciado en el modelo social de discapacidad. La cuestión relevante es la elisión de la particularidad de la problemática clínica en la afección en salud mental, deslizando la terapéutica a un tratamiento social de la discapacidad.

Habremos de coincidir en la condena a la segregación y a sostener una posición ética en el tratamiento del sufrimiento psíquico, enmarcado en el discurso de los $\mathrm{DDHH}$, pero este enfoque es insuficiente; no sin la clínica.

El psicoanálisis es una de las escasas posiciones clínicas que ha permitido el acceso, intelección y terapéutica de las afecciones psíquicas. Tampoco habremos de extendernos en este punto en el presente trabajo. Pero veamos las consecuencias de su rechazo. Por ejemplo, y por dar uno; ¿cómo se piensa la desmanicomialización, si sólo se lo considera desde la defensa de los DDHH?

¿La destitución de la lógica manicomial se alcanza solamente erradicando las instituciones monovalentes de salud mental? ¿Se trata sólo de la segregación física o edilicia o de la modificación de las condiciones de internación? En tal caso ¿se interroga sobre cuál es el estatuto de la locura y cuál su tratamiento? ¿Qué discusión se ha planteado sobre criterios de internación? ¿Qué debate se ha producido sobre la lógica de la internación y la externación, que inevitablemente ponen en juego un soporte topológico del espacio?

La locura no es el extravío de una interioridad sino desanudamiento Real, Simbólico e Imaginario; y expulsión a una exterioridad radical, donde no hay interior ni exterior, espacio ni tiempo, sólo el desamparo y en el mejor de los casos, el síntoma.

El manicomio bien puede existir a cielo abierto, en cualquier paraje de la ciudad. Remedando el Medioevo, cuando se permitía a los locos vivir extramuros, en la puerta de la ciudad, su figura actual es la tolerancia por "el loco del pueblo o del barrio". El manicomio también puede existir en un Hospital General o en el más confortable establecimiento. En la calle o en el ambiente lujoso.

Se trata de constituir instituciones no crueles, para el alojamiento y el cuidado de quién sufre, a fin de dar una oportunidad al tratamiento de su padecimiento.

Contar con dispositivos alternativos a la internación es importante. Tan importante como pensar el estatuto clínico de 
un dispositivo. La lógica de lo colectivo se distingue de la lógica de la masa. Lo colectivo es uno más uno más uno, y el lazo. Para que haya lazo, tiene que haber distancia y lugar para habitar, que aloje al viviente en su singularidad. La masa agrupa sin distancia, lo que no permite el lazo ni la singularidad del uno por uno. La oferta de dispositivos iguales "para todos" hace efecto de masa. Se piensa en términos universales elidiendo la construcción en transferencia de la respuesta singular de cada uno, que constituye disposición de espacio y tiempo.

Hospitales de día, Casas de Medio Camino, Consultorios Externos, Internación, son algunos dispositivos ofrecidos en la cura. En todo caso, bienvenida la oferta de estructuras, para que cada uno pueda servirse de ellos, o inventar el propio dispositivo a partir de la particularidad de su síntoma, en transferencia. Si no hay una construcción en la experiencia analítica en transferencia, lo producido es efecto de la sugestión y basta con que el terapeuta se aparte para que la construcción no se sostenga y se derrumbe. Sólo la construcción sinthomatica en transferencia provee un sostén propio a la ex-sistencia.

Hacer lugar a la clínica entonces. Es desde la experiencia clínica, y la impronta que produce el psicoanálisis en concordancia con otras clínicas, que se ordena el campo y se produce el paso a lo social. No hay desmanicomialización sin clínica, sin la experiencia subjetivante de cada analizante que construya ex-sistencia a partir de su naturaleza singular.

El efecto de subsumir la afección de salud mental en una discapacidad, propendiendo a un tratamiento social excluyente, ha contribuido a la elisión de la clínica. De lo cual surge una inquietante pregunta que se traslada al lector y a cada practicante de la clínica. ¿Qué orienta la práctica clínica hoy? ¿Qué orienta una práctica cuando se la enmarca y reduce al tratamiento social de la discapacidad mental?

\section{BREVE DIGRESIÓN SOBRE INTERDISCIPLINA}

La Ley 26657 en sus artículos 8 y 9 del Capítulo V, establece la condición de interdisciplina para la integración de los equipos terapéuticos. En una lectura inicial parece tratarse de una respuesta política al modelo médico hegemónico que predominó durante un largo tiempo. No obstante, quedan planteadas dos cuestiones por resolver.

Por un lado, la interdisciplina es una problemática epistémica compleja que se resuelve por la vía de la operación lógica que subtienda su articulación: complementariedad de saberes, suplementariedad o recurrencia que configuren un saber total.

A modo de axioma, diremos que el encuentro de saberes requiere de un marco preciso y de un dispositivo que permita la interlocución. Sin extendernos en desarrollos, se propone leer la interdisciplina como interdiscursividad, sostenida en la operación lógica de intersección de conjuntos. En el espacio propio de la intersección converge la falta de cada saber, el vacío, el agujero de saber. Cada saber tiende a constituirse en un saber total, todo saber; por lo cual los saberes compiten Sólo cuando es posible ponerse en falta respecto del saber constituyente, con el vaciamiento se articula el lugar para otros saberes, otros argumentos.

No se trata de abstracciones lógicas como una especulación vana. Hay expe- 
riencias que se sostuvieron en este formato lógico posibilitando el intercambio. Un ejemplo de ello son las Juntas Especiales de Dictámenes según el artículo 22 de la ley de salud mental de la Provincia de Santa Fe. Otro ejemplo, ha sido un Ateneo Clínico. En ambas experiencias lo convocante es la pregunta, el enigma, el problema sin respuesta aún. El enigma es lo que descompleta el saber e inscribe la falta que reúne y pone al trabajo.

Pero resta intacta una cuestión relevante. ¿Cómo se articula la concurrencia de saberes diversos con la necesidad de una dirección de la cura, en el caso por caso? Este interrogante ha sido desatendido, advirtiéndose en su lugar, una diversidad de malentendidos y respuestas errantes. Todos y ninguno es responsable.

\section{INTERNACIONES NO VOLUNTARIAS}

Un punto crítico son las internaciones no voluntarias que encuadramos en el contexto de la irrupción de urgencias subjetivas.

En la internación voluntaria el usuario de salud mental da su consentimiento a la internación. La internación no voluntaria procede cuando el equipo de salud mental dictamina que existe un riesgo cierto e inminente para sí o para terceros y prescinde del consentimiento de la persona afectada.

En un plazo de 48 hs debe informarse al juez competente y al Órgano de Revisión, con las fundamentaciones de la decisión terapéutica. Con un plazo de 3 días, el juez debe autorizar o denegar la internación si considera que no están dadas las causales, o solicitar informes ampliatorios.
Partamos de la premisa de que se trata de un punto muy sensible, ya que las internaciones han constituido históricamente un instrumento utilizado en ocasiones con fines segregativos, de castigo, e incluso a los fines de declaración de insanía y despojo patrimonial. El espíritu de la ley es limitar los abusos en el uso de las internaciones y brindar garantías de derecho, lo cual compartimos. Pero relevemos la experiencia institucional que hoy tiene lugar.

La primera cuestión es que definir una crisis o urgencia subjetiva requiere de una referencia clínica ineludible y sólida en sus fundamentos. Es necesario no confundir crisis con furor. La clínica psicoanalítica responde a la problemática de la crisis subjetiva con fundamentos derivados del estatuto del pasaje al acto y del acting out.

Planteamos el interrogante abierto acerca de si los practicantes de la clínica han asumido hoy la responsabilidad de dictaminar la situación de crisis, indicando la internación no voluntaria cuando una persona en crisis no está en condiciones de decidir su consentimiento, comprometiéndose en su cuidado.

Subrayemos un dato de la realidad. La mayoría de las instituciones de salud mental y servicios de urgencias médicas de la región, en mayor o menor medida, en la actualidad eluden la atención de urgencias subjetivas.

La generalidad de los jueces competentes limita su intervención a receptar pedidos de control de legalidad de las internaciones por parte de los profesionales intervinientes. No autorizan ni deniegan.

Hacemos constar que a pesar de los años transcurridos, apenas se han dado los pasos iniciales para instituir el Órgano de Revisión que respalda y da fun- 
cionamiento a la red asistencial de salud mental. Tampoco los efectores de salud mental, públicos y privados cuentan con un funcionamiento en red, que de soporte a una experiencia clínica viva en una comunidad de trabajo. Sin duda que lograr su consolidación y funcionamiento ético, constituiría un aporte decisivo.

En consecuencia, en el nivel de la existencia, se ha producido la paradoja que la persona que experimenta una crisis en salud mental, en la mayoría de las oportunidades se ve librado a sus propios medios, al desamparo y a la capacidad de respuesta de la familia, siendo la excepción la intervención comprometida de los actores concernidos.

En el mejor de los casos, si un familiar acude a un juez solicitando una medida cautelar de protección de persona, debe contar con el patrocinio de un abogado, que luego dé lugar a la evaluación forense, que debe entregar su informe al juez para que finalmente dictamine y ordene la intervención correspondiente del servicio de urgencia y de la institución asistencial. Este procedimiento puede llevar muchos días y excede largamente la pertinencia de una respuesta rápida a una crisis, que puede ser una diferencia crucial en la que se dirime la vida o la muerte de una persona.

Se pone de manifiesto que el sólo enunciado de una ley no alcanza para brindar respuestas, cuidados y garantías. Es necesario el recurso de la clínica en un contexto ético y decidido. Ello requiere de convocar a un espacio de construcción de consensos, acuerdos de trabajo y procedimientos de las partes involucradas.
¿CÓmo SE ARTICULA CLÍNICA Y POLÍTICA?

La historia de la introducción del psicoanálisis en nuestro país registra el debate sobre el modo de intersección y de articulación del discurso analítico y el discurso político, en diversos contextos y circunstancias

La articulación referida presenta una complejidad importante, abordable por la diversidad de lineamientos que aloja el espacio de intersección. Por una parte se trata de la interrogación de lo político y de la política que posibilita las elaboraciones del psicoanálisis.

Lacan conceptualiza el campo lacaniano como el campo de goce. (Lacan, 196970) A lo largo de su enseñanza encontramos indicaciones precisas que traducen las formulaciones de la economía política en términos de economía de goce. Ello interpreta la cuestión política según los modos de distribución y circulación de goce que se da una sociedad determinada. Este fundamento da lugar a una nueva perspectiva de lo político y de la política a partir del discurso del psicoanálisis.

Por otra parte, se trata de los efectos políticos que promueve el discurso analítico. En otros términos, cabe la pregunta sobre si el discurso analítico y su modo de incidencia en la subjetividad, producen consecuencias con un alcance político. Se trata de establecer si los efectos terapéuticos y éticos de la experiencia analítica cobran también un alcance político en el campo social e institucional y en la subjetividad de la época, en tanto aporta una definida dirección de la cura. A través del fin de análisis se obtiene un paso del inconsciente a lo social por la vía del sinthoma. Se especifica que el fin de análisis se presenta no sólo al final, sino también 
en el decurso de la experiencia analítica, como eso a lo que la experiencia tiende, cada vez. En esta perspectiva, Lacan llega a afirmar que la experiencia analítica posibilita una salida del discurso del capitalismo; una salida no segregativa en tanto es para cualquiera; pero es una salida singular, uno por uno.

Lacan afirma que Freud no sólo inventa el psicoanálisis y su conceptualización teórica, sino que inventa un nuevo discurso, el discurso analítico; que constituye un nuevo e inédito modo de lazo social. Un discurso que se abstiene del dominio. ¿Está justificada la formalización de una clínica del lazo social? Este interrogante, extiende las consideraciones del psicoanálisis al campo ampliado de lo social.

Como se advierte, cada pregunta presenta un anverso y un reverso discursivo que implica el campo del psicoanálisis y el campo de lo social. En consecuencia, es posible distinguir en cada interrogante un desarrollo que concierne al psicoanálisis en intensión y otro que concierne al psicoanálisis en extensión.

En su Conferencia de Milan en 1972, Lacan presenta al capitalismo con el matema de un falso discurso. Notable. Su fórmula condensa y presenta al descifrado del lector, la lógica del capitalismo; aquí en el formato de un discurso que empuja al goce en la forma del consumo anónimo. Paradoja de un embrague superyoico de goce que atenta contra su propio soporte; consumiendo se consume.

Sostendremos la hipótesis que las problemáticas de la clínica actual están atravesadas por los efectos del discurso del capitalismo y de la ciencia y la tecnología. La ciencia impone su metáfora real, entificando lo particular en el elemento-partícula, elidiendo la singularidad. El diferencial de la ciencia es el diferencial del número, desprendido de la letra y del uso singular de la letra. Es un rasgo simbólico que no cifra y que incide en la configuración de la representación de lo humano y en el lenguaje.

Verificamos en la época la dificultad de la formación de síntomas, habida cuenta de la caída de lo simbólico y de su capacidad de metáfora. En su lugar, empuje al acto, perturbación de la construcción de la espacialidad y temporalidad de la experiencia propia, del cuerpo sensible, hiperkinesis, TGD, bulimia, anorexia, toxicomanías, síndromes de atención deficitaria, violencia, impulsiones, y todo tipo de trastornos que los manuales clasifican prontamente; y configuran una nueva casuística que requiere nuevas apuestas clínicas. No se trata de promover una oposición a la ciencia, sino de producir un llamado a la consideración ética de sus efectos.

El abordaje del matema del discurso del capitalismo requiere el discernimiento de cada vector, de cada término, de cada arista, para su lectura y desciframiento. En esta oportunidad no haremos un examen detallado del discurso del capitalismo y sus efectos en la subjetividad de la época, pero señalaremos que sí constituye un tema del que los practicantes de la clínica tendríamos que hacernos cargo.

En la Conferencia de Milan, Lacan avanza aún un paso más para anunciar sin explicitarlo, un nuevo discurso que responderá a la necesidad de mantener el semblante, pero paradojalmente al servicio de lo peor. Menciona el discurso "PS", finalmente "PESTE". Un discurso apestoso al servicio del discurso del capitalismo.

Proseguimos agregando interrogantes que convocan al trabajo. ¿Qué conjetura forjar respecto del discurso "Pestoso"? 
Desarrollaremos una conjetura sobre el discurso PESTE, procediendo metodológicamente como Lacan hizo para arribar a la escritura del discurso del capitalismo, a partir de la modificación del discurso amo.

Lacan lee los efectos producidos en su contemporaneidad respecto del discurso amo, y los incorpora formalizando la escritura de su nuevo matema, el discurso del capitalismo.

Fundamentalmente lee por una parte, la caída del S1, como caída de la imago paterna, del Nombre del Padre, de los semblantes de la Ley y de lo Simbólico en su conjunto. Por otra parte, la elisión de la doble barra en el piso inferior entre el Sujeto y el goce, que nota la imposibilidad de acceso directo al goce, ahora anulada y lo imposible desamarrado.

A partir de aplicar similar metodología, haremos un ejercicio de deducción probable e hipótesis sobre el discurso "apestoso", modificando la escritura del discurso del capitalismo al incluir los efectos producidos por éste en la época.

(S1 S1 S1 S1...) Esquizia del Significante amo

(S tachado subcero) Abolición del sujeto

(S2) Saber reducido al matema de la ciencia S2

Particularización de lo viviente

Se retoma el principio del artículo, con un retorno a lo político y a lo social a partir del encuentro con la experiencia analítica y la lógica del fin de análisis. La clínica psicoanalítica conlleva no sólo un compromiso ético y terapéutico, sino también político. Ello implica sostenerse en el discurso analítico. Hacemos resonancia de la propuesta de Susana Toté, de una (a) puesta de lo "en común" que haga co- munidad de analizantes, para sostener la transmisión del psicoanálisis, en transferencia con el Discurso Analítico.

Situar la referencia política por fuera de la clínica, no solo erra de la mala manera, sino que conduce a un cambio de discurso. Del discurso analítico a los intentos de restituir el discurso amo. Ello no es sin consecuencias.

\section{REFERENCIAS BIBLIOGRÁFICAS}

Agamben, G Homo Sacer I. Editorial Nacional. (2002) Madrid. España.

Aguirre, José Luis. Hospital Psiquiátrico de Oliveros. Una Historia Revista Archivos de Medicina Familiar y General. Vol. $11 \mathrm{~N}^{\circ}$ 2. CABA: Diciembre 2014. Federación Medicina Familiar y General República Argentina. http:// archivos.famfyg.org (entrada Junio 2018)

CELS Derechos Humanos en Argentina. Informe 2012. (2012) Siglo XXI Editores. Buenos Aires (Págs. 351-376)

Ley Provincial de Salud Mental 10772/91 Internet (entrada marzo 2018) https:// www.santafe.gov.ar/index.php/web/ content/download/33098/171113/file/ Ley\%20Provincial\%2010772.pdf 
MSyMA Provincia de Santa Fe. Decreto 2155/2007 Reglamentación Ley Provincial de Salud Mental 10772/91 Internet (entrada marzo 2018) https:// www.santafe.gov.ar/normativa/item. php?id=52222HYPERLINK “https:// www.santafe.gov.ar/normativa/item. php? id $=52222 \& \operatorname{cod}=\mathrm{d} 1165789927 \mathrm{f}-$ 5235c622e1b30857be77" \&HYPERLINK "https://www.santafe.gov.ar/ normativa/item.php?id $=52222 \& \mathrm{co}-$ $\mathrm{d}=\mathrm{d} 1165789927 \mathrm{f} 5235 \mathrm{c} 622 \mathrm{e} 1 \mathrm{~b}-$ $\underline{30857 \mathrm{be} 77 " \mathrm{cod}=\mathrm{d} 1165789927 \mathrm{f}-}$ 5235c622e1b30857be77

MSyMA Provincia de Santa Fe. Resolución 660 del 7-4-2003. Reglamentación Artículo 22 Ley Provincial de Salud Mental 10772/91. Internet (entrada marzo 2018) Internet (entrada marzo 2018) https://www.santafe.gov.ar/index.php/web/content/ download/33536/172817/file/Resoluci $\%$ C3\%B3n\%200660-03.pdf

LACAN, J. La carta robada (1956) en Escritos II Editorial Siglo XXI. 1996. Mexico

El Seminario Libro X. La Identificación (1962-63) Editorial Paidós. (2006) Buenos Aires. Argentina.

El Seminario Libro XVII El reverso del psicoanálisis (1969-70) Editorial Paidós (1996) Buenos Aires. Argentina.

Seminario RSI (1974-75) (entrada junio 2018) http://www.bibliopsi. org/docs $/$ lacan $/ 27 \% 20$ Seminario $\% 20$ 22.pdf

Conferencia de Milan (1972) Internet (entrada marzo 2018) http:// www.elsigma.com/historia-viva/traduccion-de-la-conferencia-delacan-enmilan-del-12-de-mayo-de-1972/9506
RESEÑA CURRICULAR DEL AUTOR

Mario Kelman es analizante y analista. Investigador Consejo de Investigaciones UNR. Docente Facultad de Psicología UNR. Director Área Salud Mental Programa Psicoanálisis, Ciencia, Ciencia Cognitiva CEI UNR. Instituyente y Director Pasantía Clínica Facultad de Psicología UNR. Director Carrera de Posgrado de Especialización Psicología Clínica, Institucional y Comunitaria - 2005/2006. Facultad de Psicología UNR 